\title{
Online Voting Using Block Chain
}

\author{
R Tharani ${ }^{1}$, J Jereena ${ }^{2}$, Sreejith $\mathbf{P} \mathbf{K}^{3}$ \\ Assistant Professor, JCT Collge of Engineering \& Technology, Coimbatore, Tamilnadu, India ${ }^{1}$ \\ IV CSE, JCT Collge Of Engineering \& Technology, Coimbatore, Tamilnadu, India ${ }^{2,3}$
}

\begin{abstract}
Digital casting a ballot is the utilization of electronic gadgets, for example, casting a ballot machines or a web program, to cast a ballot. Security of computerized casting a ballot is dependably the greatest concern when considering executing an advanced casting a ballot framework. One way the security issues can be possibly fathomed is through the innovation of block chains. Block chain innovation begins from the fundamental engineering structure of the digital money bitcoin. It is a type of conveyed database where records appear as exchanges, a square is a gathering of these exchanges.
\end{abstract}

Keywords: Block chain, Privacy, Security, Anonymity

\section{INTRODUCTION}

Block chain is freshest developments proposed in august 2011.Block chain is the spine Technology of Digital Crypto Currency BitCoin .The block chain is a disseminated database of records all things considered or advanced occasion that have been executed and shared among partaking parties. Every exchange checked by most of members of the framework. It contains each record of every transaction. BitCoin is the most well-known cryptographic money a case of the block chain .The bitcoin is a cryptographic money and is utilized to trade computerized resources on the web. Bitcoin utilizes cryptographic confirmation rather than outsider trust for two gatherings to execute exchanges over the web. Every exchange secures through computerized signature. There is no Central Server or System which keeps the information of Block chain. The information is appropriated more than Millions of Computers around the globe which are associated with the Block chain. This framework permits Notarization of Data as it is available on each Node and is freely evident. A square is an information structure that contains all the important metadata about the block (Block Header) itself and contains exchanges. The principal hinder in a block chain is known as the beginning square. Square chain can be characterized as a chain of the square that contains data. The strategy is proposed to timestamp advanced reports with the goal that it's unrealistic to predate them or temper them. The square chain is utilized for the safe exchange of things like cash, property, contracts, and so on without requiring an outsider middle person like bank or government. When information is recorded inside a square chain, it is exceptionally hard to transform it. Square chains couldn't be kept running without the Internet. A hub is a PC associated with the Block chain Network. A chain of squares which contain some metadata about the square, a few exchanges and joined to the past square by the past square's hash esteem. Hub gets associated with Block chain utilizing the customer. Customer helps in approving and spreads exchange on to the Block chain. At the point when a PC interfaces with the Block chain, a duplicate of the Block chain information gets downloaded into the framework and the hub comes in a state of harmony with the most recent square of information on Block chain. The Node associated with the Block chain which helps in the execution of an exchange as an end-result of a motivating force is called Miners. Block chain improves trust over a business arrange. It isn't so much that you can't believe the individuals who you direct business with its that you don't have to while working on a Block chain arrange.

\section{LITERATURE SURVEY}

[1]Untangling Block chain: A Data Processing View of Block chain System Systems. Tien Tuan Anh Dinh, Rui Liu, Meihui Zhang *, Member, IEEE, Gang Chen, Member, IEEE, Beng Chin Ooi, Fellow, IEEE, and Ji Wang 2018,Block chain technologies are gaining massive momentum in the last few years. Block chains are distributed ledgers that enable parties who do not fully trust each other to maintain a set of global states. . In this paper, we survey the state of the art, focusing on private block chains. We analyze both in-production and research systems in four dimensions: distributed ledger, cryptography, consensus protocol and smart contract. [2]Block chain Reaction A block chain revolution sweeps into health care,offering the possibility for a much-needed data solution . By Leslie Mertz IEEE 2018 ,electronic health records may have digitized patient data, but getting that data from one clinician to another remains a huge challenge, especially since patients often have multiple doctors tests, prescribing drugs, and providing treatment.In addition, block chain technology might also be able to help with other aspects of health care, such as 


\section{IJARCCE}

Vol. 8, Issue 2, February 2019

improving the insurance claim or other administrative processes within healthcare networks and making health-related population data available to biomedical researchers.

[3]A survey on the security of block chain system SXiaoqiLia,PengJianga,TingChenb, XiapuLuoa,QiaoyanWencIEE E Since its inception, the block chain technology has shown promising application block chain has been applied to many fields. Although here are some studies on the security and privacy issues of block chain, there lacks a systematic examination on the security of block chains systems.[4]On Scaling and Accelerating Decentralized Private Block

chainsWei Xin ; Tao Zhang ; Chengjian Hu ; Cong Tang ; Chao Liu ; Zhong Chen IEEE 2017,Three strategies to improve the scalability of private block chain: optimization of block construction, block size and time control optimization, and transaction security mechanism optimization.

\section{METHODOLOGY}

\section{A. Create Election System}

Online Election System would have Candidate Registration, Admin Login which will be taken care of by Election Commission Candidate Login which will be dealt with By Candidate. Administrator makes a decision and notices the applicant's. Also administrator confirm User id.

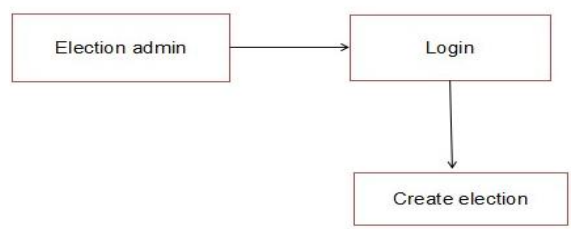

B. User Login and Give Vote

- $\quad$ Authenticated Voters login with correct username and password.

- Voter choose candidate in the election system

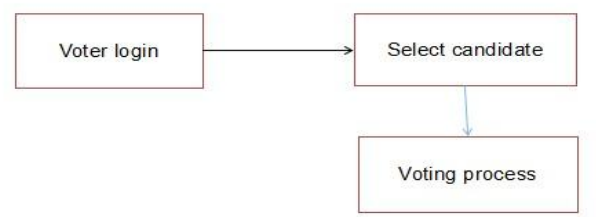

C. Create a Block Chain

- Timestamp to store the creation date of the Block.

- Hash of the previous Block.

- Vote stored in the Block.

- Hash of the current Block to ensure integrity of its content.

D. Checking the Results

By creating a block chain which keeps track of the tallies of the votes. Election admin can only eligible to view the results. The module shows the overall result who got a maximum number of votes.

\section{PROPOSED SYSTEM}

\section{Online Voting Using Block Chain}

In the proposed framework, to create e-casting a ballot framework for Lok Sabha is a reason which will have highlights of administrator and client. Here administrator will include the subtleties of Political Parties who are qualified to cast a ballot, add hopeful subtleties as indicated by the zone, and so on., User can sign in and vote to competitor and view level of votes in favor of every applicant. The proposed framework is actualized to enable every single open to effectively take part in the decision procedure. This is finished by the web application which will acknowledge the votes of various territories open utilizing the application. In this framework the voter can cast a ballot effectively by ecasting a ballot. In this security is given by squares secure database. Casting a ballot Register id is coordinated in Database and Hash esteems. In the event that the voter ID is substantial, the voter can pick the possibility to cast a ballot and furthermore check the voter id in the database.

- $\quad$ Application for block chain technology is voting.

- $\quad$ By casting votes as transactions, we can create a block chain which keeps track of the tallies of the votes.

- $\quad$ Everyone can agree on the final count because they can count the votes themselves, and because of the block chain audit trail, they can verify that no votes were changed or removed, and no illegitimate votes were added. 


\section{IJARCCE}

\section{PARTS OF BLOCK CHAIN}

A block chain is a chain of squares associated with one another. A square comprises of certain parts. Each square contains a cryptographic hash of the information of the past square. The nonce is determined by the mineworkers by tackling a cryptographic riddle to propose the following square in the chain.

A. Information: Block chain is a database or record that is shared over a system. This record is scrambled with the end goal that just approved gatherings can get to the information. Since the information is shared, the records can't be altered. Along these lines, the information won't be held by a solitary substance. It gives numerous zones of use and here we perceive how it will fill in as a superior choice to distributed storage.

B. Hash Value: A hash is a capacity that changes over a contribution of letters and numbers into a scrambled yield of a fixed length. A hash is made utilizing a calculation, and is basic to square chain the board in digital money. Hash Value is taking an info string of any length and giving out a yield of a fixed length

C. Time Stamp: The Time Stamps demonstrate that the squares are associated in a sequential request. It denotes
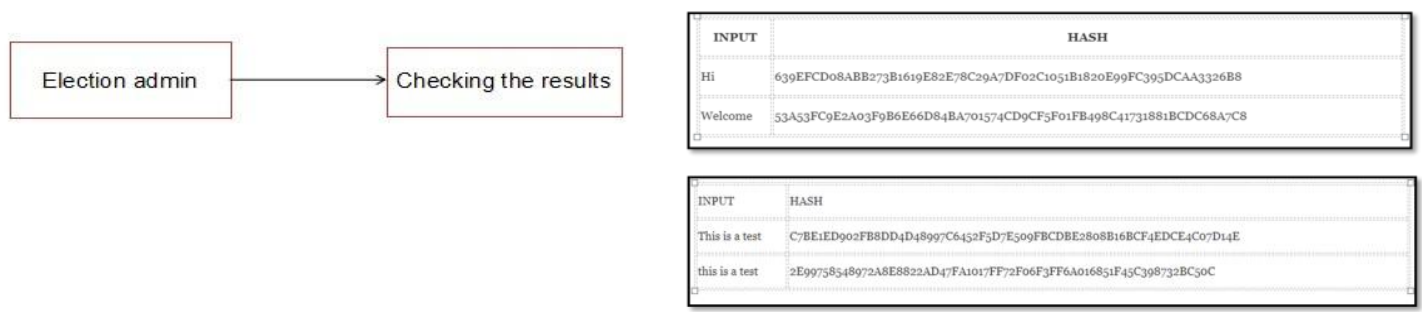

the ideal opportunity for every exchange on the block chain. The procedure of safely monitoring the creation and alteration time of an archive, is an irreplaceable instrument in the business world.

D. Nonce: A nonce is a number added to a hashed square that, when repeated, meets the trouble level limitations. Every exchange produces a hash. The nonce is the trouble. The hash depends on the exchange as well as the past exchange's hash. Indeed, even little change in a nonce the exchange makes a totally new hash.

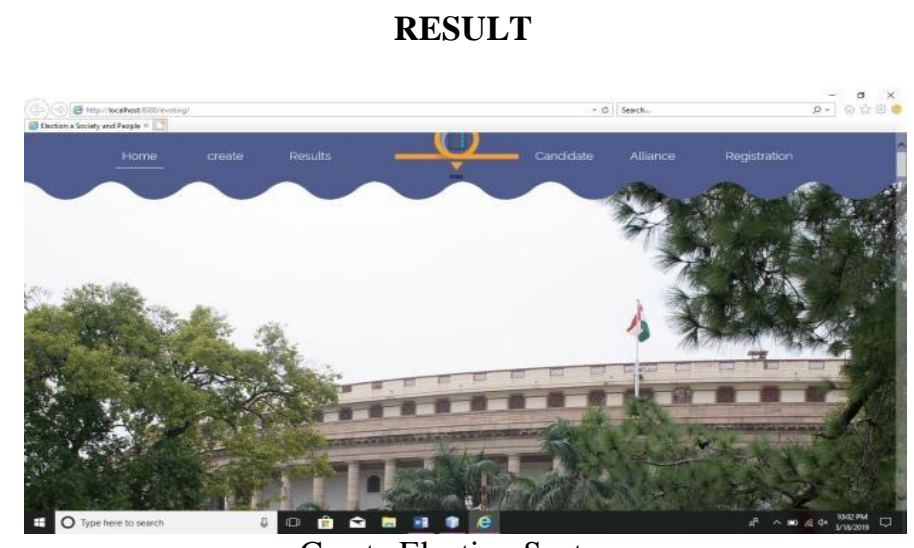

Create Election System

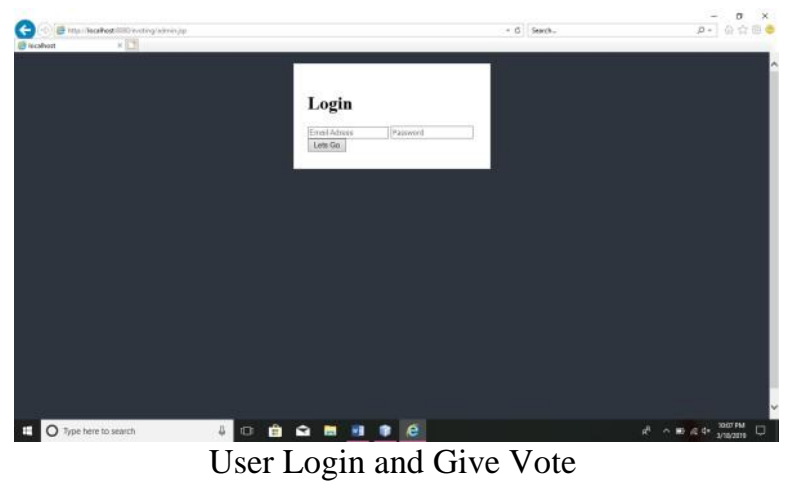




\section{IJARCCE}

Vol. 8, Issue 2, February 2019

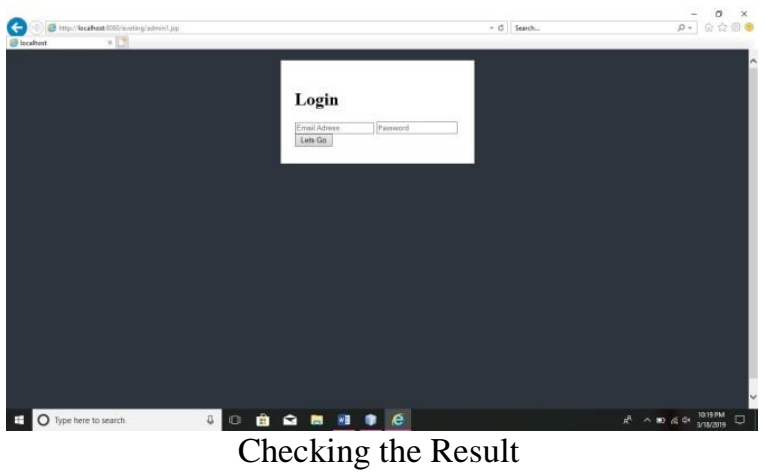

\section{ACKNOWLEDGEMENT}

We would like to express our sincere gratitude to the staff of Department Of Computer Science and Engineering, JCT College Of Engineering And Technology

\section{CONCLUSTION}

Block chain is a famous distributed digital currency and decentralized system. Bitcoin utilizes cryptographic evidence rather than outsider trust for two gatherings to execute exchanges over the web. Every exchange secures through advanced mark. There is no Central Server or System which keeps the information of Block chain. The information is appropriated more than Millions of Computers around the globe which are associated with the Block chain. This framework permits Notarization of Data as it is available on each hub and is openly unquestionable. A square is an information structure that contains all the fundamental metadata about the block(Block Header) itself and contains transactions. It give more grounded obscurity and protection. At long last, we investigate the convention in all regards.

\section{FUTURE SCOPE}

The web based casting a ballot framework stage can be made increasingly secure by utilizing following strategies secret phrase changing, finger printing and cornea recognition. No outsider access in block chain and progressively decentralized shared money arrange.

\section{REFERENCES}

[1] Tien Tuan Anh Dinh, Rui Liu, Meihui Zhang *, Member, IEEE, Gang Chen, Member, IEEE, Beng Chin Ooi, Fellow, IEEE, and Ji Wang 2018.

[2] Block chain Reaction A block chain revolution sweeps into health care,offering the possibility for a much-needed data solution.. By Leslie Mertz IEEE 2018

[3] A survey on the security of block chain system sXiaoqiLia,PengJianga,TingChenb,XiapuLuoa,QiaoyanWenc IEEE 2018

[4] On Scaling and Accelerating Decentralized Private Block chains. Wei Xin ; Tao Zhang ; Chengjian Hu ; Cong Tang; Chao Liu ; Zhong Chen IEEE 2017

[5] Y. Yuan and F. Wang, "Parallel block chain: Concept, methods and issues," Acta AutomaticaSinica, vol. 43, no. 10, pp. 1703-1712, 2017.

[6] A. Kosba, A. Miller, E. Shi, Z. Wen, and C. Papamanthou, "Hawk: The block chain model of cryptography and privacy-preserving smart contracts," in Proc. IEEE Symp. Security Privacy (SP), 2016, pp. 839-858.

[7] K. Christidis and M. Devetsikiotis, "Block chains and smart contracts for the Internet of Things," IEEE Access, vol. 4, pp. $2292-2303$, 2016.

[8] R. N. Z. Aitzhan and D. Svetinovic, "Security and privacy in decentralized energy trading through multi-signatures, block chain and anonymous messaging streams," IEEE Trans. Depend. Secure Comput., vol. 15,no. 5, pp. 840-852, Sep./Oct. 2016.

[9] F. Santoso,"A new framework for rapid wireless tracking verifications based on optimized trajectories in received signal strength measurements,” IEEE Trans. Syst., Man, Cybern., Syst., vol. 45, no. 11,pp. 1424-1436, Nov. 2015. 